\section{Christoph Bachmann}

Die Mariendistel (S. marianum (L.) Gaertn., früher: Carduus marianus) ist eine ein- bis zweijährige, bis $150 \mathrm{~cm}$ hohe Pflanze, deren Verbreitungsgebiet der östliche Mittelmeerraum ist (Abb. 1). Die Mariendistel ist auch von Südrussland bis in den Iran anzutreffen. Ihr Name stammt von einer alten Legende, nach der die weissen Flecken auf den Blättern von der Milch der Jungfrau Maria stammen.

\section{Inhaltsstoffe, Pharmakologie}

Hauptinhaltsstoff von S. marianum ist Silymarin, das selbst ein Gemisch aus vier Flavonolignan-Isomeren ist. Die biologisch aktivste Form von diesem Gemisch ist Silibinin (Abb. 2). Die hauptsächliche Wirkung von Silymarin besteht in der antihepatotoxischen Stabilisierung der Zellmembran. Weiter stimuliert es die Regenerationsfähigkeit der Hepatozyten. Silymarin behindert das Eindringen von toxischen Noxen und den Verlust von Zellbestandteilen wie Transaminasen. Dies geschieht durch eine Verminderung der Oxidationsund Transportprozesse in der Zell-

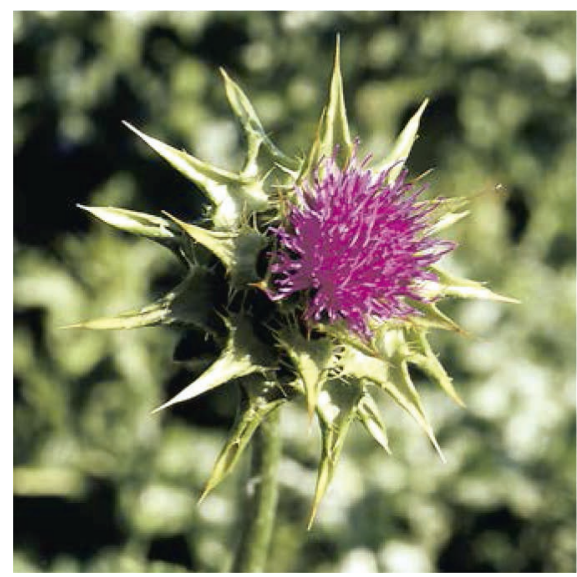

Abb. 1. Mariendistel (S. marianum).

\title{
Mariendistel zur Behandlung verschiedener Leberschäden: Klinische Studien belegen die Wirksamkeit
}

Die Arzneipflanze Mariendistel (Silybum marianum) besitzt den Gallenfluss fördernde und hepatoprotektive Eigenschaften. Man kann sie auch bei schweren Leberschäden wie Hepatitis, Chemotherapie-induzierter Hepatotoxizität, Amatoxin-Vergiftungen sowie bei nichtalkoholischer Fettlebererkrankung (non-alcoholic fatty liver disease (NAFLD)) einsetzen, wie die folgenden klinischen Studien und Erfahrungsberichte zeigen.

membran. Weiter stimuliert Silymarin die Bildung neuer Hepatozyten [1]. Die Radikalfänger-Eigenschaften von Silymarin scheinen dabei eine wichtige Rolle zu spielen [2].

\section{Klinik}

S. marianum wird schon seit etlichen Jahren klinisch erforscht. Es gibt eine Reihe älterer und verschiedene neuere Studien, die die klinische Wirksamkeit untersuchten. Eine Zusammenstellung wichtiger älterer Studien findet man in der Übersichtsarbeit von Iten et al. [2]. In den letzten Jahren wurden unter anderem Studien publiziert, die die Wirksamkeit der Mariendistel bei Chemotherapiebedingter Hepatotoxizität bei Kindern, bei Pilzvergiftung, bei verschiedenen Hepatitis-Arten sowie bei nichtalkoholischer Fettlebererkrankung erforschten.

\section{Chemotherapie-bedingte Hepatotoxizität}

Die Chemotherapie bei Patienten mit Tumorerkrankungen hat oft eine leberschädigende Nebenwirkung, die z.B. im Falle von an akuter lymphoblastischer Leukämie (ALL) erkrankten Kindern zum Therapieabbruch führt. Dies ist vor allem während der<smiles>COc1cc([C@H]2Oc3cc(C4Oc5cc(O)cc(O)c5C(=O)[C@H](O)C4CO)ccc3OC2CO)ccc1O</smiles>

Abb. 2. Silibinin.

Erhaltungsphase der Behandlung der Fall. Da immer mehr Berichte über eine günstige Wirkung von Mariendistel-Extrakten bei solchen Leberschädigungen auftauchten, führte ein Autorenteam um Elena Ladas eine entsprechende Studie [3] durch, in der die klinische und die In-vitroWirksamkeit untersucht wurden, hier aber nur der klinische Teil resümiert wird.

Bei dieser 28 Tage dauernden doppelblinden, randomisierten, placebokontrollierten Studie wurden 50 Patienten zwischen 1 und 21 Jahren, die an ALL litten, eingeschlossen. 26 von ihnen wurden in die Placebo-Gruppe randomisiert und 24 in die VerumGruppe, die während 28 Tagen Kapseln mit $240 \mathrm{mg}$ Mariendistel-Extrakt erhielten, der auf $80 \mathrm{mg}$ Silibinin A und B standardisiert war. Die Dosis wurde auf 5,1 mg/Extrakt/kg Körpergewicht berechnet. An den Tagen 28 (Behandlungsende) und 58 wurden in beiden Studiengruppen die Transaminase- und die Bilirubin-Konzentrationen gemessen, die bei Baseline zwischen den beiden Gruppen keine

\section{KARGER}

Fax +497614520714 Information@Karger.com www.karger.com (c) 2014 S. Karger GmbH, Freiburg 
statistisch signifikanten Unterschiede aufwiesen. Am Tag 28 bestand zwischen den beiden Studiengruppen keine signifikant tiefere Konzentration an Amino-Alalin-Transferase (ALT) und Aspartat-Amino-Transferase (AST). Am Tag 58 wurde jedoch in der Mariendistel-Gruppe gegenüber der Placebo-Gruppe eine signifikant tiefere Konzentration an AST ( $p=0,05)$ sowie ein Trend $z u$ einer signifikant tieferen Konzentration an ALT ( $p=0,07)$ ermittelt.

Bei den beiden Behandlungsgruppen konnten keine signifikanten Unterschiede in Bezug auf berichtete unerwünschte Ereignisse festgestellt werden.

Aus diesen Resultaten schlossen die Autoren, dass der verwendete Mariendistel-Extrakt bei ALL eine wirksame und sichere Supportiv-Behandlung sein kann.

\section{Chronische Hepatitis C}

An der Universität im iranischen Isfahan wurde von einem Forscherteam um Hamid Kalantari eine Studie durchgeführt [4]. Es handelte sich um eine selbstkontrollierte, d.h. also offene, Studie, bei der 55 Patienten eingeschlossen wurden, die folgenden Kriterien entsprachen: Anwesenheit einer diagnostizierten chronischen Hepatitis C (HCV Ab $\{+\}$, HVC-RNA (mit PCR) $\{+\}$ ) mit normalen oder erhöhten Leberenzymen (ALT und AST) ohne gleichzeitige Medikation mit Interferon oder Ribavirin. Die Patienten wurden während 24 Wochen mit $630 \mathrm{mg}$ Silymarin/Tag (einem standardisierten iranischen Arzneipräparat) behandelt; vor und nach der Behandlung wurden verschiedene Parameter wie ALT, AST, HCV-RNA (mit PCR) und mehrere Marker für Leberfibrose erhoben. Gemäss den Werten dieser LeberfibroseMarker wurden die Probanden bei Baseline in drei Gruppen eingeteilt: i) Fibrose-Gruppe; ii) NichtfibroseGruppe; iii) Intermediäre Gruppe.
Sowohl bei den ALT- als auch bei den AST-Werten zeigte sich nach den 24 Behandlungswochen eine statistisch signifikante Verbesserung der Werte gegenüber Baseline (ALT: $\mathrm{p}<0,001 ;$ AST: $\mathrm{p}=0,004)$. Nach Behandlungsende wurden bei 9 Patienten keine HCV-RNA mehr gefunden. Bei den Fibrose-Markern wurde nur in der Fibrose-Gruppe eine statistisch signifikante Verbesserung ermittelt. Die von den Patienten angegebene Lebensqualität verbesserte sich durch die Behandlung signifikant ( $\mathrm{p}<0,001)$.

Damit konnte durch diese Studie belegt werden, dass die Behandlung mit 630 mg Silymarin/Tag bei Patienten mit chronischer Hepatitis C wirksam ist.

\section{Akute Hepatitis}

El-Kamary et al. [5] publizierten 2009 eine Studie über die Wirksamkeit von Silymarin bei akuter Hepatitis. Bei dieser randomisierten, placebokontrollierten Doppelblindstudie wurden 105 Patienten im Alter von >13 Jahren (Durchschnitt: 29,8 Jahre) mit typischen Symptomen einer akuten klinischen Hepatitis und Serumwerten von ALT > 100 IE/l, mit den äusserlich sichtbaren Anzeichen einer Hepatitis und mit drei oder mehr der folgenden Symptome eingeschlossen: dunkelgelber Urin, leicht gefärbter Stuhl, Fieber, Nausea, Erbrechen, Anorexie, Aversion gegen Rauchen, Pruritus, Beschwerden im oberen rechten Abdominalbereich, Druckbeschwerden sowie juckender, roter Hautausschlag seit weniger als 4 Wochen.

Die 105 Probanden wurden in die Verum-Gruppe $(\mathrm{n}=55)$ oder in die Placebo-Gruppe $(n=50)$ randomisiert und erhielten während 4 Wochen dreimal täglich entweder $140 \mathrm{mg}$ eines standardisierten Mariendistel-Präparates (das Präparat ist in der Schweiz als Legalon ${ }^{\circledR}$, Rottapharm SA, im Handel) oder Placebo. Nach weiteren 4 Wochen wurde eine weitere Visite durchgeführt. An den Tagen 2, 4 und
7 sowie 2, 4 und 8 Wochen nach Baseline wurden die Symptome einer akuten Hepatitis überprüft sowie Leberfunktionsprüfungen durchgeführt.

Bei den Patienten der VerumGruppe stellte sich eine schnellere Verbesserung der auf biliäre Retention zurückführenden Symptome, d.h. dunkler Urin ( $p=0,013)$, Gelbpigmentierung der Haut $(p=0,02)$ und Sklerenikterus ( $p=0,043)$, sowie eine Verminderung des indirekten Bilirubins ein. Bei den anderen erhobenen Parametern konnte zwischen der Verum- und der Placebo-Gruppe kein signifikanter Unterschied festgestellt werden. Daraus schlossen die Autoren, dass eine Behandlung der akuten Hepatitis mit dem verwendeten Mariendistel-Extrakt die Symptome dieser Erkrankung verbessern kann. Es fällt auf, dass bei dieser Studie das Mariendistel-Präparat oral verabreicht wurde. Trotzdem konnte ein positives Resultat festgestellt werden. Dies liegt an der Tatsache, dass es sich hier nicht um eine chronische Hepatitis C, sondern um eine akute Hepatitis handelt, die oft auch auf eine orale Therapie mit Mariendistel anspricht.

\section{Vergiftungen durch Amatoxin}

Etwa 90\% aller weltweit auftretenden Pilzvergiftungen sind auf Amatoxin zurückzuführen, ein Octapeptid, das unter anderem im Knollenblätterpilz (Amanita phalloides) enthalten ist und einige Stunden nach der Einnahme Abdominalkrämpfe, Erbrechen sowie eine Cholera-ähnliche Diarrhö bewirkt und zu Nieren- und Leberversagen mit tödlichen Folgen führen kann.

Mengs et al. [6] publizierten 2012 eine Übersichtsarbeit, in der sie Studien und Fallberichte vorstellten, bei denen Patienten mit einer Amatoxin-Vergiftung mit Legalon ${ }^{\circledR}$ SIL, einem standardisierten MariendistelExtrakt, behandelt wurden. Aus ethischen Gründen gibt es hierfür keine 
kontrollierten klinischen Studien, dafür jedoch eine Fülle von unkontrollierten Studien und Fallberichten, bei denen die Patienten intravenös mit Legalon SIL behandelt wurden (Legalon ist in der Schweiz nur in einer oral anwendbaren Form zugelassen). Bei diesen etwa 1500 dokumentierten Fällen verringerte sich die Mortalitätsrate auf weniger als 10\%. Bei einer Behandlung mit Penicillin oder einer Kombination von Penicillin und Silibinin, einem Inhaltsstoff der Mariendistel, betrug die Todesrate $>20 \%$. Es konnte gezeigt werden, dass Silibinin antiinflammatorische und antioxidative Eigenschaften hat und mit spezifischen Leber-Transportproteinen interagiert und so die intrazelluläre Wiederaufnahme von Amatoxin verhindert. Dies bewirkt einen Unterbruch des enterohepatischen Kreislaufs der Toxine, was auch bei Patienten mit schwerster AmatoxinVergiftung noch hilfreich sein kann. Dabei spielte die Zeit zwischen der Amatoxin-Einnahme und dem Beginn der Mariendistel-Behandlung eine entscheidende Rolle. Wenn die Patienten innerhalb von $48 \mathrm{~h}$ behandelt wurden, erwies sich der Verlauf der Lebervergiftung meistens als eher leicht bis mittel. Wenn die Patienten jedoch erst später Hilfe erhielten, dann wurden oft viel schwerere Leberschäden beobachtet. Aus diesen zusammengetragenen Studien und Fallberichten schlossen die Autoren, dass das Mariendistel-Präparat Legalon das Mittel der Wahl bei akuter Amatoxin-bedingter Lebervergiftung ist.

\section{Nichtalkoholische \\ Fettlebererkrankung}

Butorova et al. [7] untersuchten in einer offenen, randomisierten Parallelgruppen-Studie die Wirksamkeit von Legalon ${ }^{\circledR}$ bei NAFLD verschiedener Genese. Dafür wurden 70 Patienten mit einer diagnostizierten NAFLD in die Studie aufgenommen. Für die NAFLD-Diagnose wurde eine Steatose mit Ultraschall festgestellt. Die Patienten wurden in zwei Gruppen (beide $\mathrm{n}=35$ ) randomisiert:

- Legalon-Gruppe: Diese Patienten erhielten während 3 Monaten dreimal täglich $140 \mathrm{mg}$ Legalon.

- Kontrollgruppe: Bei diesen Patienten wurde eine nichtmedikamentöse Therapie durchgeführt: Diät zur Gewichtsreduktion, regelmässige körperliche Betätigung).

Beide Gruppen wurden in zwei Untergruppen eingeteilt: i) Patienten mit Fettdystrophie (FD) der Leber; ii) Patienten mit nichtalkoholischer Steatohepatitis (NASH).

Als Wirksamkeitskriterien wurden klinische (Allgemeinzustand, Müdigkeit, Schmerzen in der Lebergegend, Lebergrösse), biochemische (ALT, AST, GGT (Gamma-Glutamyl-Transferase)) und Ultraschall-Parameter (Veränderung der Lebergrösse) bestimmt.

Alle Patienten der Legalon-Gruppe beendeten die Therapie und berichteten grossmehrheitlich (92\%) von einer subjektiven Verbesserung des Allgemeinbefindens. In der Kontrollgruppe konnte keine solche subjektive Verbesserung festgestellt werden. 2 Patienten berichteten sogar von einer subjektiven Verschlechterung des Allgemeinbefindens.

In der Legalon-Gruppe wurde eine signifikante Verminderung der Transaminasen-Aktivität ermittelt. Bei allen Patienten (100\%) der FDGruppe stellte sich eine Normalisierung der Transaminasen ein. In der NASH-Subgruppe war dies bei $80 \%$ der Patienten der Fall. Bei den übrigen $20 \%$ verbesserten sich die Werte vom Dreifachen auf das Anderthalbfache des Normalwertes. Alle Patienten der Legalon-Gruppe wiesen eine Normalisierung der GGT auf.

In der Legalon-Gruppe konnte bei 4 Patienten (40\%) der FD-Subgruppe sowie bei 5 Patienten (20\%) der NASH-
Gruppe eine Verkleinerung des Lebervolumens festgestellt werden.

Die Legalon-Behandlung wurde von allen Patienten ohne Nebenwirkungen gut toleriert.

Daraus schlossen die Autoren, dass Legalon ein wirksamer und sicherer Hepatoprotektor im Falle von NAFLD ist und zu einer signifikanten Verbesserung von klinischen und biochemischen Parametern führt.

\section{Fazit}

Die hier vorgestellten Studien dokumentieren die Wirksamkeit standardisierter Mariendistel-Extrakte bei Leberschäden verschiedener Genese. Daher kann diese Arzneipflanze bei Chemotherapie-bedingter Hepatotoxizität, bei Hepatitis, bei Amatoxin-bedingter Pilzvergiftung sowie bei NAFLD als ideales Therapeutikum empfohlen werden. Es zeichnet sich durch eine gute Wirksamkeit und durch die sehr geringe Anzahl an Nebenwirkungen aus.

\section{Literatur}

$>1$ Wellington K, Jarvis B: Silymarin; a review of its clinical properties in the management of hepatic disorders. Bio Drugs 2001;15:465-489.

2 Iten F, et al: Silymarin in der Behandlung von Lebererkrankungen. Phytotherapie 2003;3: 18-24.

3 Ladas EJ, et al: A randomized, controlled, double-blind, pilot study of milk thistle for the treatment of hepatotoxicity in childhood acute lymphoblastic leukemia (ALL). Cancer 2010;116:506-513.

4 Kalantari H, et al: Effects of silybum marianum on patients with chronic hepatitis. J Res Med Sci 2011;16:287-290.

5 El-Kamary SS, et al: A randomized controlled trial to assess the safety and efficacy of silymarin on symptoms, signs and biomarkers of acute hepatitis. Phytomedicine 2009;16:391-400.

6 Mengs U, et al: Legalon ${ }^{\circledR}$ SIL: the antidote of choice in patients with acute hepatoxicity from amatoxin poisoning. Curr Pharm Biotechnol 2012;13:1964-1970.

7 Butorova LI, et al: Potential for the use of Legalon ${ }^{\circledR}$ in non-alcoholic fatty liver disease (Artikel in russischer Sprache). Eksp and Klin Gastroenterol 2010;3:85-91. 